Cahiers $d u$ MONDE RUSSE

\section{Cahiers du monde russe}

Russie - Empire russe - Union soviétique et États indépendants

$55 / 3-4 \mid 2014$

Varia

\title{
Alexander Etkind, Warped Mourning, Stories of the Undead in the Land of the Unburied
}

\section{Georges Nivat}

\section{OpenEdition}

Journals

Édition électronique

URL : http://journals.openedition.org/monderusse/8142

DOI : $10.4000 /$ monderusse. 8142

ISSN : $1777-5388$

Éditeur

Éditions de l'EHESS

\section{Édition imprimée}

Date de publication : 1 juillet 2014

Pagination : $511-517$

ISBN : 978-2-7132-2441-6

ISSN : $1252-6576$

\section{Référence électronique}

Georges Nivat, «Alexander Etkind, Warped Mourning, Stories of the Undead in the Land of the Unburied », Cahiers du monde russe [En ligne], 55/3-4 | 2014, mis en ligne le 14 avril 2015, Consulté le 24 septembre 2020. URL : http://journals.openedition.org/monderusse/8142 ; DOI : https://doi.org/ 10.4000/monderusse.8142

Ce document a été généré automatiquement le 24 septembre 2020.

(c) École des hautes études en sciences sociales 


\title{
Alexander Etkind, Warped Mourning, Stories of the Undead in the Land of the Unburied
}

\author{
Georges Nivat
}

\section{RÉFÉRENCE}

Alexander ETKIND, Warped Mourning, Stories of the Undead in the Land of the

Unburied, Stanford : Stanford University Press (Cultural Memory in the Present), 2013, $320 \mathrm{p}$.

1 Cette fois-ci le nouvel ouvrage d'Alexander Etkind contient des paramètres personnels. "Ce livre est enraciné dans l'histoire de ma famille », explique l'historien des idées, professeur à l'université européenne de Florence. L'ouvrage est dédié à son père, l'historien d'art Mark Etkind, et à son oncle, l'historien de la littérature européenne, chef de file d'une grande école de traduction poétique, Efim Etkind. Leur père fut arrêté dans son appartement de Saint-Pétersbourg en 1930. "Cinq mois plus tard son fils rentrait de l'école, et il demanda à un vieillard grisonnant qui était assis dans l'escalier : « je peux passer, s'il vous plaît?». C'était le père de Efim et de Mark, et le petit garçon Efim ne reconnut son père que lorsque celui-ci s'adressa à lui par son petit nom familier : «Fima !». Grigorij Etkind avait survécu à cinq mois d'horrible promiscuité, à de multiples tortures. Il avait entendu l'annonce de sa prochaine exécution, avait été fourré dans un fourgon puis jeté sur le trottoir devant sa propre maison. Quelque chose qui ressemblait à la mise en scène de l'exécution de Dostoevskij en 1849. Grigorij avait vieilli de vingt ans en cinq mois. Plus tard l'oncle disait au neveu : «Ce que j'ai entendu raconter par mon père était plus terrible qu'Une terrible vengeance de Gogol'. »Cette comparaison avec le plus "gothique » des textes de Gogol' joue un grand rôle dans le déploiement du livre dédié à sa famille par Alexander Etkind. 
2 Le livre s'intitule étrangement " Warped Mourning », ce qu'on peut traduire en français par «Un deuil biaisé », ou encore déformé, comme une pièce de bois qui a " gauchi ». Écrit un demi-siècle après les confidences de l'oncle Fima, il a mis la distance qui, selon le théoricien Stephen Greenball, est nécessaire pour que la littérature se distancie d'un tragique passé, ici la catastrophe du stalinisme, du Goulag, de l'automassacre de la Russie pendant la période communiste. Soit deux générations culturelles.

3 C'est le livre de Jacques Derrida, Spectres de Marx (1993) qui a visiblement servi de point de départ aux réflexions d'Etkind. Derrida y définit comme spectrale toute l'Europe révolutionnaire. Le spectre étant celui que mentionne le Manifeste communiste dès la première phrase : «Un spectre hante l'Europe, le communisme ». Mais Marx lui-même devint un spectre, et l'utopie marxienne, sa déformation catastrophique, et les réactions à cette déformation sont comme un échange spectral, une circulation de revenants, une « hantologie » (on trouve le mot chez Derrida).

4 Les paroles du spectre de son père à Hamlet sur les remparts d'Elseneur sont réemployées tant par Derrida que par Etkind.

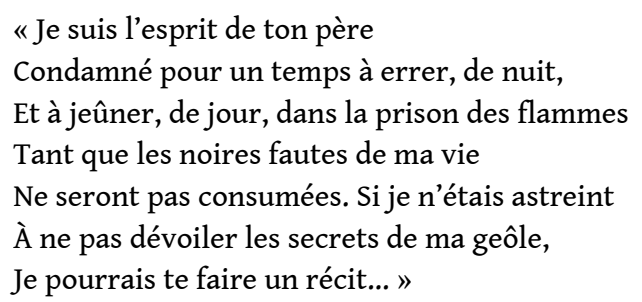

5 Le secret dont parle le fantôme du livre d'Etkind, c'est le non-deuil de la Russie pour son crime du Goulag, un crime perpétré sur elle-même. Et, comme le dit Derrida, il n'est guerre pire que l'intestine, la "civile», comme on dit. L'ouvrage est à la fois une randonnée dans la Russie d'aujourd'hui qui coïncide en partie avec celle que j'ai faite moi-même ces dernières années : Medvežegorsk au nord de la Carélie, la ville nouvelle au nord du lac d'Onega où se dresse encore aujourd'hui l'énorme building de l'ancienne administration du Belomorkanal, à trente kilomètres de Povenec, point où le canal long de $225 \mathrm{~km}$ aboutit au lac Onega. Etkind y rend visite au directeur, et nous conduit à une quinzaine de kilomètres de là, en pleine forêt, le lieu découvert par Mémorial où quelques milliers de bagnards venus du camp de concentration de Solovki, sur l'archipel de la mer Blanche furent fusillés en trois jours. Le lieu fut retrouvé grâce un ancien capitaine Nkavédiste, qui dirigeait les exécutions à la chaîne, mais plus tard arrêté lui-même avait survécu et témoigné. Sandarmoh est effectivement un des lieux de mémoire les plus extraordinaires de la Russie actuelle, quoique fort peu fréquenté : des milliers de troncs d'arbres portent des photos des victimes, punaisées par les descendants ou survivants, comme si la forêt elle-même était devenue un ossuaire, un mémorial. Il y a en Russie très peu de monuments aux victimes du Goulag, qui sont pourtant millions, présentes presque dans chaque famille. Etkind décrit succinctement tous ces lieux, en particulier les deux énormes pierres venues de Solovki et qui témoignent par leur force tellurique, l'une à Moscou, l'autre à Saint-Pétersbourg.

Cette rareté des monuments, et leur ancrage symbolique et minéralogique dans la pierre de Solovki, qui pendant cinq siècles fut l'avant-poste nordique de l'orthodoxie russe, prison monastique puis prison bagne de 1921 à 1939, pose un problème de mémoire. Etkind pense que la paucité des monuments de pierre dévoile la difficulté à mettre un terme au deuil, à enterrer les morts qui, n'étant pas morts reviennent comme fantômes et vampires dans la vie inconsciente de la Russie d'aujourd'hui. Il 
élargit d'ailleurs le thème, de façon assez simpliste, à toute la littérature russe depuis Gogol' et son "récit gothique » Une terrible vengeance. Ce côté "roman gothique », avec revenants et sautes d'un extrême à l'autre de l'hystérie dénonciatrice à l'autoaccusation impudique, apparait comme une sorte d'invariant tant de la culture que de l'histoire russe. Un chapitre s'intitule " Mourning and Warning ", soit « Deuil et avertissement ", analyse de ce qu'est le deuil et de ce qu'est le traumatisme (en s'appuyant sur un texte de Freud, en 1914 - Remémoration, répétitions et perlaboration ${ }^{1}$. Lorsque le deuil n'est pas accompli, le traumatisme s'enracine dans l'inconscient, personnel ou collectif, passe de génération en génération et l'expérience originelle du traumatisme se répète de façon compulsive. Cette "compulsion répétitive " serait le principal symptôme de la maladie russe d'aujourd'hui. Etkind oppose d'une part la précision chirurgicale des Mémoires de Nadežda Mandel'štam, qui savait très exactement QUI elle avait perdu, en la personne d'Osip, le poète tout clairvoyant, et d'autre part les millions de Soviétiques qui avaient perdu des leurs, mais ne savaient pas pourquoi, ni qui ils avaient vraiment perdu (un délateur? un indic? une victime prise au hasard? un authentique conjuré ?). Lidija đukovskaja a magnifiquement décrit cette seconde et immense catégorie dans son récit « Sof'ja Petrovna » (aussi connu sous le titre La maison désertée).

7 Etkind parcourt de son faisceau explicatif toute la littérature russe dite dissidente comme toute la culture soviétique. Cela va de Sinjavskij à Sorokin ou Šarov aujourd'hui. Sans employer le mot, il décrit Sinjavskij comme une sorte de marrane soviétique, cachant son antisoviétisme, qui prend racine dans une foi révolutionnaire originelle. Etkind rappelle cette scène extraordinaire de Bonne nuit où le fils assiste au délire du père qui, rentré du camp, se croit «branché » sur les écoutes du KGB même au milieu d'une épaisse forêt où il a entrâné son fils. D'où la culpabilité du fils, qui s'exprime à travers tous ses ouvrages du camp et d'après le camp. L'" affaire des historiens", montée en 1929, et qui a donné lieu à 150 arrestations, nous montre également le « marranisme » des survivants qui, comme Tarlé, écrivent une biographie de Napoléon, appréciée par Stalin, et récompensée du Prix Stalin au sortir du goulag; ou encore l'historien Družinin dont le livre sur les serfs d'État semble tracer une sorte de projet datant de Nicolas Ir $^{\text {er }}$ de ce qui sera la collectivisation à la soviétique. Ou encore l'historien Boris Romanov, dont la vie et l'œuvre ont été écrits par Viktor Panejah, et dont l'ouvrage principal, Hommes et mours de l'ancienne Russie est accusé de misanthropie, mais ne fut pas mis au pilori.

De façon un peu inattendue mais convaincante, l'académicien Dmitrij Lihačev apparaît comme une figure centrale dans le livre d'Etkind: l'auteur de tant d'ouvrages sur la culture et la littérature de la Russie médiévale a lui-même raconté à la fin de sa vie comment il a commencé sa carrière scientifique par une étude in situ sur le jargon des voleurs russes, étudiés au camp de Solovki où il avait été déporté, comme des milliers d'intellectuels et d'ecclésiastiques, à la fin des années 1920. C'était une époque où les arrestations étaient encore "compréhensibles »: le jeune Lihačev faisait partie d'un groupe estudiantin qui s'intitulait l'Académie cosmique des sciences. Et sa première publication parait dans la revue du camp, Soloveckie ostrova, en 1930 ; elle a pour titre : "Les jeux de cartes des condamnés de droit commun (étude du cabinet criminologique) ». Lihačev gagna la confiance des truands, et le jeune philologue conclut de son étude que leur jargon n'était pas une langue codée pour se cacher des autres, comme on le croyait habituellement, mais une langue ludique, une résistance 
aux malheurs de la vie par le rire. Le rire sera d'ailleurs le sujet d'un de ses derniers livres.

9 Etkind le compare à Mihail Bahtin, lequel, au même moment, élaborait ses ouvrages sur le carnavalesque, d'abord chez Dostoevskij, puis chez Rabelais. Le carnavalesque, qui a conquis le monde intellectuel américain et en est devenu une part de la doxa, tout comme la déconstruction derridienne, est lui aussi une autodéfense, mais Etkind note que Bahtin a fort bien su se camoufler dans la société soviétique. Lihačev, lui, à la fin de sa vie, est devenu une sorte de guide intellectuel national à l'époque gorbatchévienne.

Tous ne plient pas sous le faix d'un deuil inachevé ; il y a ceux qui font comme si rien ne s'était passé et reviennent de leur bagne inchangés. Le shakespearologue Leonid Pinskij ${ }^{2}$ ne changea pas une ligne à son ouvrage, bien qu'il semblât que l'étude de Shakespeare ait dû considérablement s'enrichir dans le topos du goulag, shakespearien presque par définition, c'est-à-dire fait d'extrêmes opposés. Mais ici intervient, selon Etkind la "nostalgie de la culture mondiale », dont Mandel'štam est un des porteurs les plus géniaux, mais qui a véritablement soutenu une large part de l'intelligentsia russe pendant l'épreuve de sa réduction en sujétion et soumission. On peut d'ailleurs s'étonner, dans la poursuite de telles réflexions, que le régime n'ait pas vraiment décidé de supprimer " les classiques », comme le fit plus logiquement Mao au paroxysme de sa révolution culturelle. Peut-être fut-ce une erreur fatale pour le communisme stalinien ? Bien entendu censure et mise à l'écart de livres dans les enfers des bibliothèques furent pratiquées, mais l'essentiel de ce que la culture russe avait élaboré sous l'ancien régime, en résistance à cet ancien ordre des choses, fut conservé et pouvait aisément être utilisé contre les nouveaux maîtres. Le pouchkiniste Oksman, un des responsables de l'édition jubilaire de Puškin pour le centenaire de 1937, fut dénoncé comme saboteur dudit centenaire, fit dix ans de camp, survécut, reprit ses études sur le contexte politique et social de Puškin, ainsi que ses déchiffrements textuels des manuscrits. Etkind rappelle comment Oksman introduisit une variante du texte de la célèbre lettre de Belinskij à Gogol', variante qui ne parut que bien plus tard et qui, en soi, était un acte de dissidence tant du poète que du textologue. En 1963, Oksman fit passer en Occident son texte vengeur "Délateurs et traîtres parmi les écrivains et les savants soviétiques ». Autre cas, Eleazar Meletinskij, un des plus importants ethnographes, commença ses études au camp, et y découvrit que chacun se raccrochait à son ethnie.

Deux artistes ont droit à de fort intéressants portraits, un peu disproportionnés dans l'économie générale du livre. Il s'agit tout d'abord du peintre Boris Svečnikov, un des rares artistes à avoir caché et rapporté du camp des œuvres (dessins à la plume pour l'essentiel). Un important lot d'œuvres de Svečnikov se trouve en Amérique au Zimmerli Art Museum à New Brunswick, mais il faut ajouter que la France en détient aussi, grâce au musée de Grenoble. Goya est présent en filigrane dans les dessins fantastiques de Svečnikov, mais un autre détenu de la mort, le croate Music, me semble avoir apporté un témoignage plus profond, plus brutal, en tout cas moins esthétisant sur l'homme réduit au statut de déchet. Etkind a raison de mettre Svečnikov à côté des résistants par la «nostalgie de la culture ». Svečnikov avait été arrêté très jeune, à 19 ans, et revint de camp en 1953, pour être réhabilité trois ans plus tard. Il s'établit à Tarusa, qui fut une petite capitale d'anciens zeks artistes ou poètes (Anastasija Cvetaeva s'y établit à son retour du goulag). Etkind analyse plusieurs tableaux de Svečnikov, dont un assez énigmatique, représentant deux jeunes hommes qui se retournent pour se dévisager, et qui sont nus sous des blouses attachées par-derrière, 
laissant voir leurs fesses. Je me demande s'il ne faudrait pas voir là une allusion au grand maître des Templiers et son adjoint, qui furent accusés de sodomie, et brûlés vifs en 1314, à Paris. Ce qui ne ferait que conforter la thèse de la « nostalgie pour la culture mondiale » développée par Etkind.

Le deuxième artiste est le réalisateur de cinéma Kozincev, un juif de Kiev. Le cas est particulièrement intéressant depuis que sa veuve a publié ses Notes et journaux. Il avait lié amitié au goulag avec Pinskij, Oksman, et le philologue de Leningrad Gukovskij qui, lui, mourut à la prison de Lefortovo à Moscou. Kozincev a eu une longue carrière, commencée dans les années 1920 , avec son célèbre film Le manteau qui, comme tous les travaux des formalistes de l'époque ignore le final fantastique du récit de Gogol' : le fantôme qui revient arracher les manteaux aux riches seigneurs qui parcourent la ville la nuit. En fait on peut penser que c'est le thème de la vengeance sociale qui était ainsi censuré. En 1939 il travaille sur un film consacré à Marx, mais ne parvint pas à mener à bien le projet. Etkind parle d'un double deuil vécu par le marxiste Kozincev pendant le dégel : pour les victimes du régime et pour les idées de Marx. Kozincev survécut à tout, et revint en force avec ses films shakespeariens de 1969, Hamlet, et de 1970, Lear. Dans Hamlet, joué par le jeune acteur prodige Smoktunovskij, est rendu une sorte d'hommage à Mihoëls, l'acteur juif tué pendant la campagne anticosmopolite, qui avait déjà incarné Hamlet. Le Hamlet de Kozincev, nous montre Etkind, est un acte de vengeance qui n'aurait pu être créé ailleurs que dans l'URSS des années 1948-1949. Pour Etkind, comme pour la critique Turovskaja, qu'il cite, c'est une exigence du droit à la complexité. Les longues scènes de l'enterrement de Hamlet, qui correspondent à quelques lignes de Shakespeare, sont selon Etkind un deuil enfin réussi pour les années de la Terreur.

13 Le cinéma plus récent est, lui aussi, longuement évoqué par l'auteur du livre, en particulier le cas de Hrustalëv, ma voiture! (1998) un film par Aleksej German dont le père était un chantre de la police politique soviétique. L'auteur analyse finement le jeu entre remémoration et imagination du narrateur : l'arrestation du père, général en vue, brusquement projeté en enfer, jeté dans un camion où des truands le sodomisent sans fin, puis abandonné sur un bas-côté, est une scène insupportable. Mais ne relève-t-elle pas davantage du thème invariant de la cruauté russe, étudié par l'historien Anisimov, que du deuil inachevé pour des morts qui ne sont pas vraiment morts? Deux films de Pavel Lungin sont également longuement étudiés: L'île, ou la naissance d'un thaumaturge, et surtout Tsar. On peut, contrairement à Etkind, interpréter l'épisode central comme la naissance d'un saint (le métropolite Philippe, livré par le tsar aux mains de son bourreau). Ajoutons que depuis le cinéaste Lungin a signé une pétition de l'intelligentsia russe en faveur du président Putin et de l'annexion de la Crimée. Quant à Maljuta, le bourreau d'Ivan, il mérite à lui seul une étude beaucoup plus vaste car il est un des grands thèmes récurrents de la littérature russe. "Le peuple russe est comme un enfant qui rêve d'un père sévère ", a dit Lungin dans une interview récente.

On ne peut que souscrire à une des conclusions du livre: il y a plus en Russie aujourd'hui de monuments littéraires et immatériels (sur le net), que de monuments en pierres consacrés au génocide intérieur que fut le goulag. À ce sujet Etkind formule une critique des Lieux de mémoire de Pierre Nora, comme si cet ouvrage considérable et novateur s'était principalement attaché aux monuments de pierre. Il n'en est rien, et le lecteur des huit tomes de l'ouvrage sait qu'il porte autant sur les monuments intellectuels, ou de l'histoire des mœurs. Tony Judt, le grand critique du New York 
Review of Books, cité ici par Etkind, avait tort de comparer cet ouvrage aux grands monuments de Mitterrand, telle la Pyramide du Louvre.

Le livre d'Etkind s'achève sur la littérature contemporaine, et ses auteurs favoris qui sont Pelevin et Sorokin. Le chapitre dit qu'outre le software et le hardware (les monuments tangibles et le musée virtuel créé par Memorial), il existe une troisième mémoire actuellement en Russie, littéraire essentiellement, et qui correspond à ce que Jacques Derrida baptise l'Hantologie, le retour des fantômes. Nous sommes hantés par des monstres, bien plus que ne l'était le Moyen-Âge des gargouilles de Notre-Dame de Paris, les monstres de Jurij Mamleev, les insectes de Pelevin, les blocs de chair gelée de Sorokin. Dans un roman de Šarov, Avant et alors, madame de Staël, devenue immortelle, partage son sort avec l'auteur illuminé de la fin du XIX siècle, Fëdorov, qui croyait en la résurrection de tous les morts, et dont l'œuvre influença Majakovskij autant ou plus que Mamleev ou Šarov.

C'est donc un ouvrage foisonnant que ce nouveau livre d'Alexandre Etkind. Il entraîne dans des perspectives éclairées par Freud ou plus encore Derrida. Il fait passer dans son rapide projecteur toute l'histoire culturelle soviétique, le cinéma soviétique, la dissidence, les postmodernistes actuels, qui connaissent tant de faveur aux États-Unis. Dans sa conclusion, il parle de réalisme magique. Et l'applique aux postmodernistes d'aujourd'hui. Je ne suis pas sûr que Pelevin fasse le même travail de guérison que Marquez... Quant à la notion de deuil inachevé, elle n'est pas vraiment élaborée jusqu'au bout. Elle est applicable universellement: le deuil islamiste de la longue soumission à l'Occident colonialiste peut expliquer la vague actuelle de violence. L'ouvrage est passionnant, mais sa direction n'est pas évidente.

17 Cependant le livre du neveu de Efim Etkind, comme il aime à se nommer lui-même, prolonge une réflexion de son oncle sur culture et anticulture dans le totalitarisme soviétique. Tout n'était pas anticulture et la culture servait de refuge, y compris dans les isolateurs du KGB. Si Thermidor il y a eu, comme disait Trockij dès la fin des années 1920, avant de recevoir le coup de piolet de l'envoyé du grand «thermidorien », c'est un thermidor plus complexe, je crois, que celui que voudrait exprimer Sorokin. Et d'ailleurs les vrais thermidoriens, ceux qui survécurent à la guillotine et se mirent à danser, n'étaient pas si malheureux que cela...

Ajoutons que le livre d'Etkind se place sous les auspices du «Bal des Victimes» qu'organisèrent les parents de victimes guillotinées dès le lendemain de la mort de Robespierre. Cela devint le rendez-vous des merveilleuses et des gandins, on se coiffait "à la victime ", les cheveux bien relevés, la nuque dégagée comme pour aller sous la guillotine. Les bals des victimes de l'époque du Directoire peuvent-ils servir de modèle à la société russe en mal de son deuil après les soixante-dix ans de totalitarisme et les dizaines de millions de victimes enterrées clandestinement dans ces milliers de Sandarmoh? De toute façon, la Terreur jacobine n'était, comme le dit Sarah Kofman, que «l'enfance de l'art». 


\section{NOTES}

1. Traduction due à Agnès Ouvrard et Gudrun Römer, sur le site Lutecium, site freudien et lacanien.

2. P. Lepin [Leonid Pinskij], «Paraphrases et hommages », Sintaksis : publicistika kritika polemika (Fontenay-aux-Roses), 1981, p. 113-69. Choix de textes précédé d'une courte introduction], choix de textes, traduction et introduction par Georges Nivat.

\section{AUTEURS}

\section{GEORGES NIVAT}

Université de Genève 